\title{
Fabrication of subwavelength structured surfaces via electrospray deposition of nanobeads
}

\author{
M. Heilmann \\ heilmama@hs-weingarten.de \\ M. Pfeffer
}

Y. Yamagata

\section{S. Morita}

University of Applied Science Ravensburg-Weingarten, Weingarten, 88250, Germany

University of Applied Science Ravensburg-Weingarten, Weingarten, 88250, Germany

RIKEN Laboratories, VCAD Applied Fabrication Team, Wako, 351-0198, Japan

The usability of electrospray deposition (ESD) to form antireflection structured (ARS) surfaces was studied experimentally. Subwavelength structured (SWS) surfaces were produced by depositing nanobeads with diameters of $50 \mathrm{~nm}$ onto glass substrates covered with an ITO-Film. The reflectance from the substrates could be decreased about nearly 3\% with an onesided ARS surface. [D0I: 10.2971/je0s.2010.10023]

Keywords: electrospray deposition, antireflection structured surfaces, subwavelength structured surfaces

\section{INTRODUCTION}

Antireflection structured surfaces (ARS) are playing an important role in the fields of the applied optics, where high transmittance is desired, this can be the case for solar cells. If light is incident on the surface of such a cell, a part of it gets reflected due to the higher refractive index of it ( $n_{\mathrm{S}} \approx 1.5$ for glass). But at the surfaces of those cells, reflectance is undesirable because it lessens the intensity of the transmitted light, and therefore the energy output. To suppress this reflectance one can apply a subwavelength structured (SWS) surface [1]. Such a surface has a grating period which is smaller than the wavelength of the incident light ( $350 \mathrm{~nm}$ to $800 \mathrm{~nm}$ for the visible light) and can be produced through electron beam lithography, an expensive and time consuming method.

A cheaper way to fabricate SWS surfaces could be the electrospray deposition (ESD) [2]. During the ESD, a high voltage is applied to a solution which is than atomized and deposited on a grounded substrate. The ESD has the advantage that it can be conducted at normal pressure and at room temperature. So maybe ESD generated SWS surfaces have the possibility to replace current methods.

\section{THEORY}

SWS surfaces can best be described by considering a linear grating with a period $\Lambda$ in one dimension from which light is diffracted into several directions (see Figure 1). For a small enough period of the grating $\Lambda \ll \lambda$, only the zeroth-order diffracted light waves are transmitted and reflected and all higher orders are evanescent. If this is the case, light propagates through the grating as it would through a coating where the refractive index $n_{\text {eff }}$ is changing with the depth and thus with the filling factor. The grating equation can be calculated to see if an order propagates or not [3],

$$
n \sin \theta_{m}-n_{0} \sin \theta_{i}=\frac{m \lambda}{\Lambda}
$$

where $n_{0}$ is the refractive index of the incident medium, $n$ is the refractive index of the medium in which the diffracted orders propagate ( $n_{0}$ for reflectance and $n_{\mathrm{S}}$ for transmittance), $\theta_{m}$ is the angle of $\mathrm{m}$-th order and $\theta_{i}$ is the angle of incident, measured from the grating normal. Since only the zeroth order should propagate, the period to wavelength dependence, or the normalized grating period, should be

$$
\frac{\Lambda}{\lambda} \leqslant \frac{1}{n_{\mathrm{s}}+\sin \theta_{\max }}
$$

where $\theta_{\max }$ is the maximum angle of incidence for which reflection should be suppressed. This is only valid for $n_{0}=1$. The denominator of Eq. (2) is always greater than its numerator, so that the period should be smaller than the wavelength of the incident light.

Compared to flat AR coatings, the SWS surfaces have the advantage that they suppress reflection over a broad spectrum and for different angles of incident $[4,5]$. The diffraction of periodic SWS surfaces can be simulated by using the effective medium theory (EMT) [6] or the rigorous coupled-wave analysis (RCWA) [7].

\section{EXPERIMENTAL METHOD}

For the creation of the SWS surfaces ESD was utilized. During the ESD electrostatic forces between a capillary and a substrate are used to form very small particles out of a solution which is in the capillary [8]. If a high voltage is applied to it the solution within is drawn to the substrate, which leads to the 
grating normal

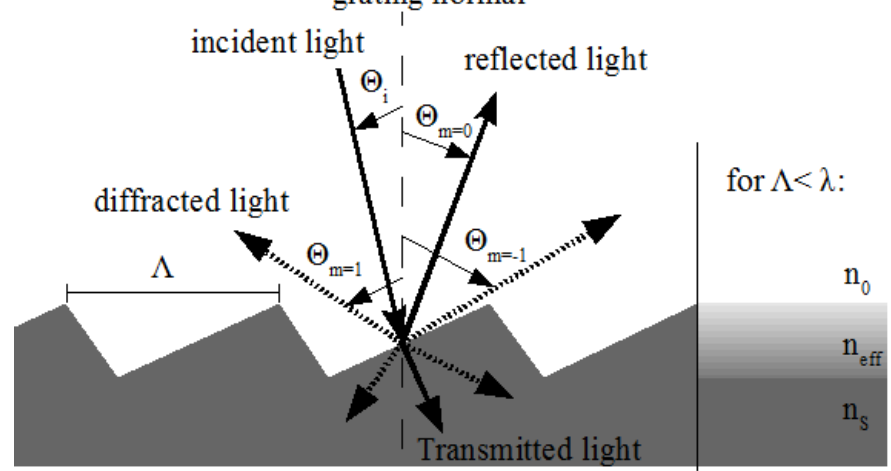

FIG. 1 Diffraction by a linear grating. Light is incident on a grating and diffracted along several paths. For $\Lambda<\lambda$, the grating will behave like a coating with a refractive index changing from $n_{0}$ to $n_{\mathrm{s}}$.

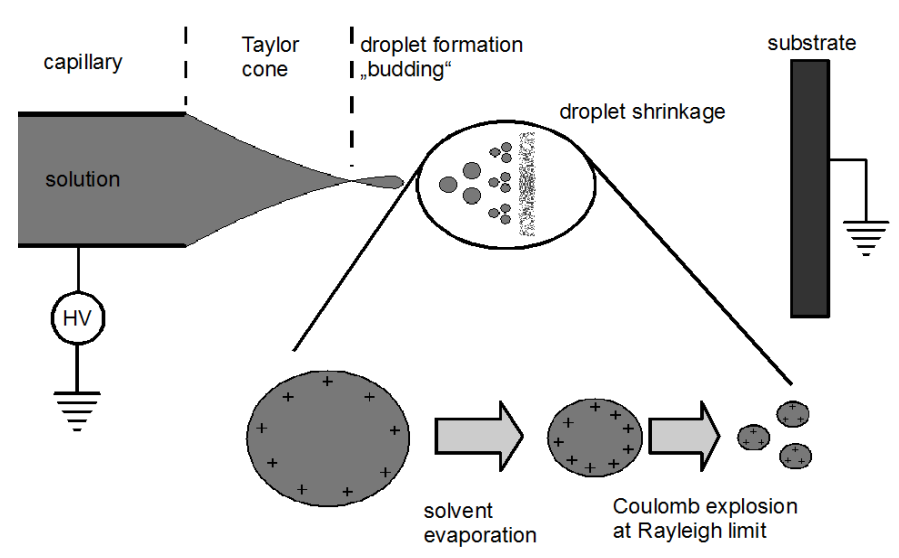

FIG. 2 For the electrospray deposition (ESD), a high voltage is applied between a solution in a capillary and a substrate. Due to electrostatic forces, solvent evaporation and Coulomb explosion, very small particles can be deposited onto the substrate.

formation of a Taylor cone (see Figure 2). At a critical voltage the attractive forces between the solution and the substrate exceed the surface tension of the Taylor cone and charged droplets are formed, this is the so called "budding". While the droplets are drawn to the substrate the solvent evaporates and the charges within the droplets converge, leading to repulsive forces between them. Coulomb explosion occurs if those forces exceed the surface tension of the droplet (at the Rayleigh limit) and very small particle diameters can be achieved [9].

The SWS surfaces were produced by ESD of nanobeads with diameters of $50 \mathrm{~nm}$ and a refractive index of 1.59 (Micromer ${ }^{\circledR}$ ). For the deposition, the ESD Arrayer from Fuence was used and the nanobeads were purchased from Micromod Partikeltechnologie $\mathrm{GmbH}$. The nanobeads were dispersed in water in concentrations between $0.1 \%$ and $1 \%$. The solutions were poured into a glass capillary with a tip diameter of $50 \mu \mathrm{m}$ and deposited onto a glass substrate, which was covered with a thin ITO-Film to make the normally insulating surfaces conducting. The distance between the capillary and the substrate was $3 \mathrm{~cm}$ and the humidity in the chamber was varying between $42 \%$ and $54 \%$. For the deposition, a voltage between $3 \mathrm{kV}$ and $4 \mathrm{kV}$ had to be applied, depending on the humidity and the concentration of the solution.

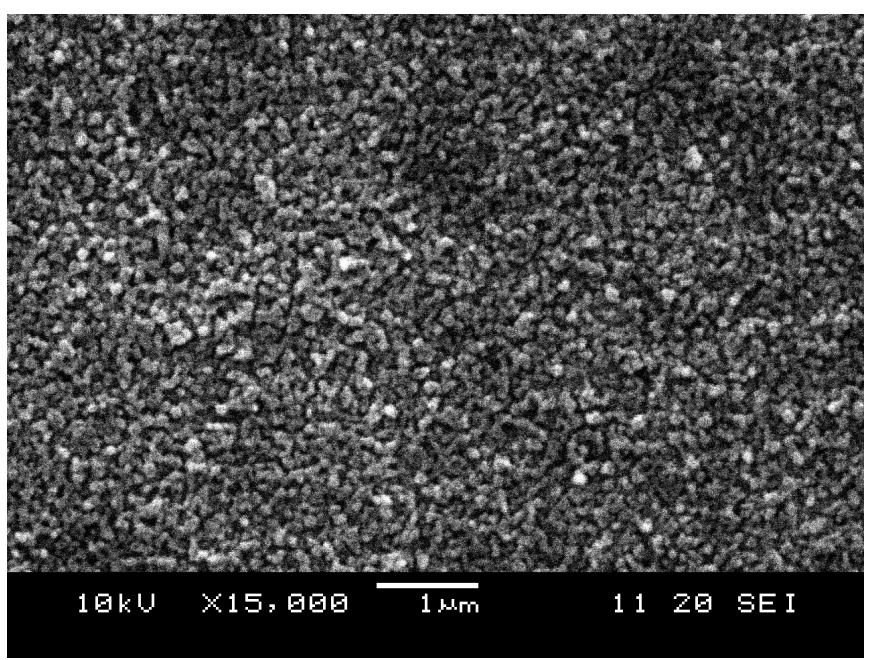

FIG. 3 SEM image of a glass surface where $2.36 \mu \mathrm{L}$ of Micromer ${ }^{\circledR}$ solution were deposited.

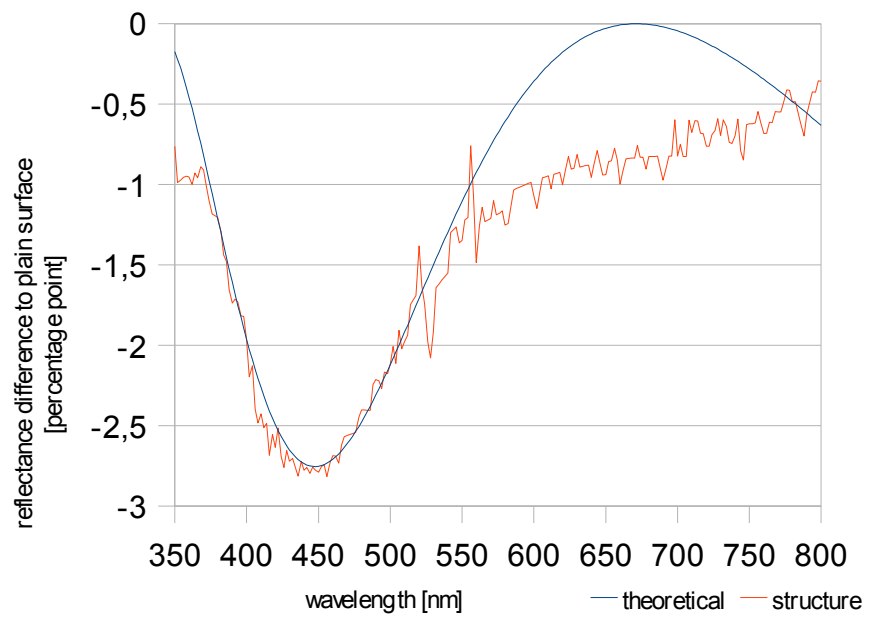

FIG. 4 Comparison between the reflectance difference of a structured to a plain surface and a calculated difference of a $245 \mathrm{~nm}$ thick AR coating with a refractive index of 1.37 to a plain glass surface.

\section{RESULTS AND DISCUSSION}

The structure from Figure 3 was produced by depositing $2.36 \mu \mathrm{L}$ of a $0.5 \%$ Micromer® solution onto a glass substrate covered with an ITO-Film. A voltage of $3.62 \mathrm{kV}$ was applied at a humidity of $50 \%$.

The difference in the reflectance of the structure from Figure 3 to a plain glass surface $\left(n_{\mathrm{S}}=1.5\right)$ was measured to be around $2.5 \%$ for wavelengths between $400 \mathrm{~nm}$ and $500 \mathrm{~nm}$. This was compared to the difference calculated for an antireflection coating with a refractive index of 1.37 and a thickness of $245 \mathrm{~nm}$ (see Figure 4). We didn't use the RCWA or EMT because the structures we obtained were neither periodical nor did they have a constant depth. The parameters for the coating were altered such that the graph of the theoretical coating was fitted to the measured reflectance of the structured surface. For wavelengths below $500 \mathrm{~nm}$, the structure acted nearly like the calculation for the AR coating, whereas for greater wavelengths the reflectance of our structure was 
smaller. This could indicate that our structure behaves like a SWS surface for wavelength above $500 \mathrm{~nm}$.

\section{CONCLUSION}

We have shown that ESD can be a simple application for the fabrication of SWS surfaces on conductive substrates. Since the time of deposition and the costs for the materials was very low, this method could be a cheap alternative to existing ones. Maybe the fabrication method can in the future be used to improve the performance of solar cells or in the entertainment industry to apply ARS surfaces to flat panel displays in order to lessen reflectance from their surface and get a clearer picture for a smaller prize. It is advisable to further investigate the structure through atomic force microscopy in order to gather evidence about the structure depth and about the distances within the structure. Thus one can compare the structures to calculations with the RCWA or the EMT.

\section{References}

[1] A. Gombert, W. Glaubitt, K. Rose, J. Dreibholz, B. Bläsi, A. Heinzel, D. Sporn, W. Döll, and V. Wittwer, "Subwavelength-structured surfaces on glass" Thin Solid Films 351, 73-78 (1999).
[2] A. Jaworek, "Electrospray droplet sources for thin film deposition" J. Mater. S. 42, 266-297 (2007).

[3] D. H. Raguin, "Analysis of antireflection-structured surfaces with continuous one-dimensional surface profiles" Appl. 0pt. 32, 25822598 (1993).

[4] W. H. Southwell, "Pyramid-array surface-relief structures producing antireflection index matching on optical surfaces" J. Opt. Soc. Am. A 8, 549-553 (1991).

[5] C. Heine, "Submicrometer gratings for solar energy applications" Appl. 0pt. 34, 2476-2482 (1995).

[6] E. B. Grann, "Optimal design for antireflection tapered twodimensional subwavelength grating structures" J. Opt. Soc. Am. A 12, 333-339 (1995).

[7] M. G. Moharam, "Formulation for stable and efficient implementation of the rigorous coupled-wave analysis of binary gratings" J. Opt. Soc. Am. A 12, 1068-1076 (1995).

[8] S. J. Gaskell, "Electrospray: principles and practice" J. Mass Spectrom. 32, 677-688 (1997).

[9] I. B. Rietveld, "Electrospray deposition, model, and experiment: Toward general control of film morphology" J. Phys. Chem. B 110, 23351-23364 (2006). 